Original Article - Clinical Science

\title{
Association of long-term glycaemic control on tear break up times and dry eye symptoms in Chinese patients with type 2 diabetes
}

Andre Ma BA(Cantab), ${ }^{3}$ Martin SY Mak, Kendrick Co Shih FCOphth(HK), ${ }^{1,2}$ Claudia KY Tsui MSc, ${ }^{3}$ Rachel KY Cheung, ${ }^{3}$ Sherman Haynam Lee, ${ }^{3}$ Hubert Leung, ${ }^{3}$ Joyce NS Leung, ${ }^{3}$ Jason TH Leung, ${ }^{3}$ Marco Zhenji Van-Boswell, ${ }^{3}$ Michael TL Wong, ${ }^{3}$ Alex Lap-Ki Ng FRCS, ${ }^{1,2}$ Chi-Ho Lee FHKCP, ${ }^{4,5}$ Vishal Jhanji FRCS ${ }^{6}$ and Louis Tong FRCS PhD $7,8,9,10$

1. Department of Ophthalmology, Li Ka Shing Faculty of Medicine, The University of Hong Kong, 301B, Cyberport 4, 100 Cyberport Road, Pokfulam, Hong Kong SAR

2. Lo Fong Siu Po Eye Centre, Grantham Hospital, 125 Wong Chuk Hang Road, Hong Kong SAR

3. Li Ka Shing Faculty of Medicine, The University of Hong Kong, 21 Sassoon Road, Pokfulam, Hong Kong SAR

4. Department of Medicine, Li Ka Shing Faculty of Medicine, The University of Hong Kong, 21 Sassoon Road, Pokfulam, Hong Kong SAR

5. KK Leung Diabetes Centre, Queen Mary Hospital, 102 Pokfulam Road, Hong Kong SAR

6. Department of Ophthalmology, University of Pittsburgh School of Medicine, Pittsburgh, Pennsylvania, United States of America

7. Ocular Surface Research Group, Singapore Eye Research Institute, Singapore

8. Corneal and External Eye Disease Service, Singapore National Eye Centre, 11 Third hospital Avenue, Singapore

9. Eye-Academic Clinical Program, Duke-NUS Medical School, Singapore

This is the author manuscript accepted for publication and has undergone full peer review but has not been through the copyediting, typesetting, pagination and proofreading process, which may lead to differences between this version and the Version of Record. Please cite this article as doi: $10.1111 /$ ceo.13146

This article is protected by copyright. All rights reserved. 
10. Department of Ophthalmology, Yong Loo Lin School of medicine, National University of Singapore, Singapore

\author{
Correspondence: Dr. Kendrick Co Shih Department of Ophthalmology, Li Ka Shing \\ Faculty of Medicine, University of Hong Kong, 301B Cyberport 4, 100 Cyberport Road, \\ Pokfulam, Hong Kong SAR. \\ Email: kcshih@hku.hk
}

Short running title: Blood glucose control and dry eye in diabetics

Received 7 September 2017; accepted 8 January 2018

Conflict of interest: None

Funding sources: None

All authors attest that they meet the current ICMJE criteria for authorship. 


\section{ABSTRACT}

Importance: Diabetes mellitus is known to be associated with dry eye syndrome (DES), but the effects of long term glycaemic control on tear film metrics and dry eye symptoms are not known in the Chinese population.

Background: To evaluate tear film stability and dry eye symptoms and their associations with systemic risk factors in Chinese patients with type 2 diabetes (T2DM) Design: Cross-sectional study set at the Lo Fong Siu Po Eye Centre (Grantham Hospital), Hong Kong.

Participants: 80 Chinese participants, aged 18 or above, with T2DM recruited from the specialist outpatient setting were included.

Methods: The Oculus Keratograph 5M (Oculus Inc, Germany) was used to measure the non-invasive tear break-up time (NITBUT). Ocular symptoms were evaluated using the Ocular Surface Disease Index (OSDI). The association between OSDI, NITBUT and metabolic parameters relating to diabetes were evaluated using multiple linear regression.

Main Outcome Measures: The associations between long term glycaemic control and NITBUT and OSDI scores.

Results: Stepwise multiple linear regression analysis revealed $\mathrm{HbA1c}$ to be the only significant independent variable for NITBUT $\left(R^{2}=0.099, P=0.014\right)$, and OSDI $\left(R^{2}=0.062\right.$, $\mathrm{P}=0.044$ ) after controlling for potential confounders. The age-adjusted prevalence of DES was $20 \%$ (95\% CI: $11-30 \%$ ) in the Chinese T2DM population. The odds of DES for increasing percentage of HbA1c was 1.49 (95\% CI: 1.03-2.17, $\mathrm{p}=0.04$ ).

Conclusions and Relevance: Our findings highlight the importance of good glycaemic control as a modifiable risk factor for both dry eye symptoms and tear film instability in patients with T2DM. 
Keywords: diabetes mellitus; dry eye syndrome; prevalence; Chinese population; glucose control

This article is protected by copyright. All rights reserved. 


\section{INTRODUCTION}

Type 2 diabetes mellitus (T2DM) is a complex and challenging chronic disease that has become a global pandemic, threatening the health and economy of all nations. In the past century, Asia's rapid economic development has made it the epicentre of the health crisis. In Hong Kong, it is estimated that one in ten people have T2DM - a number that is expected to increase with our aging population. ${ }^{1}$ T2DM and its complications are known to be a major cause of mortality and morbidity. Among the many organs affected by T2DM, the involvement of the cornea is often underappreciated, yet, it is an important area of focus because severe ocular surface disorder can be sight threatening and thus affecting all aspects of life and productivity. ${ }^{2}$ It has been well established that patients with diabetes have an increased risk of developing dry eye syndrome (DES). ${ }^{3,4}$ The Asia Dry Eye Society has recently defined DES as "a multifactorial disease characterised by unstable tear film causing a variety of symptoms and/or visual impairment, potentially accompanied by ocular surface damage" ${ }^{5}$ According to epidemiological studies, the global prevalence of dry eye is estimated to be $14.5 \%$, with the prevalence rate in Hong Kong around $7.7 \%$ in $2011 .^{6,7}$ Hence, DES is an important public health problem that calls for prevention, early diagnosis and management. The potentially debilitating symptoms of dry eye, along with its high prevalence, impose a substantial economic and social burden on the society. A study by Yu et al. calculated the annual cost of managing DES to be around $\$ 3.84$ billion for the U.S. healthcare system. ${ }^{8}$ The adverse effects of DES on health-related quality of life are also evident - two studies showed that utility scores for severe DES are comparable to those reported for moderate to severe angina. ${ }^{9,10}$ 
For patients with T2DM, the prevalence of DES among western populations is up to $54.3 \%$, which has consistently been shown to be higher than that among the corresponding general population. ${ }^{11,12}$ However, there is currently no comparable dataset in the Hong Kong Chinese populations where the overall prevalence of T2DM is around $10 \%$, and increasing up to $20 \%$ in those aged over 65 years. ${ }^{1,13}$ Studies have shown that, for the same body mass index (BMI), Asians have a higher prevalence of T2DM than the western populations. ${ }^{14}$ There is further evidence that the incidence of T2DM is rapidly increasing in the region, highlighting the increasing importance of studying DES among Chinese patients with T2DM. ${ }^{15-17}$ Diabetes predisposes to dry eye and impaired corneal epithelial wound healing. ${ }^{18-24}$ However, while several studies have found correlations between certain diabetic parameters and the risk of developing DES, the underlying mechanisms responsible for this is not yet fully understood. ${ }^{25-27}$ In particular, the ocular and systemic factors affecting tear stability or short tear break up type of dry eye is not known, which is important considering that the short tear break up type of dry eye is the most common type of dry eye in Asia. ${ }^{28}$ The aim of our study was to investigate the association between non-invasive tear film break up times, dry eye symptoms and systemic risk factors in patients with T2DM.

\section{METHODS}

The study protocol was approved by the Institutional Review Board of the University of Hong Kong/Hospital Authority Hong Kong West Cluster (HKU/HA HKW IRB).

\section{Subject recruitment}

A cross-sectional study was conducted in Hong Kong from January to March 2017. Eighty consecutive Chinese patients with T2DM mellitus between 36 and 90 years of age were recruited from the Queen Mary Hospital. Inclusion criteria were as follows: 
aged 18 or above, Chinese ethnicity, diagnosed with T2DM. Patients who suffered from any active corneal diseases were excluded. Consecutive potential study subjects were approached individually by the investigators. The study procedure, possible risks, and options for withdrawal at a later date were communicated clearly before informed consent was obtained in writing from each participant. Study participants were also provided with their own copy of the study information sheet, as well as relevant contacts, for later referral. Figure 1 displays the flowchart of the study procedure.

\section{Tear film metrics}

Tear film metrics assessment and standardised questionnaires were administered to all 80 eligible subjects at the Lo Fong Siu Po Eye Centre (Grantham Hospital). Tear film metrics were assessed with the Oculus Keratograph $5 \mathrm{M}$ to evaluate tear meniscus height (TMH) and non-invasive tear break-up time (NITBUT) for both eyes of each subject. The mean value of three measurements was taken for each corneal parameter. Ocular symptoms were evaluated via the validated Ocular Surface Disease Index (OSDI), Standard Patient Evaluation of Eye Dryness (SPEED), and a visual analogue scale Symptom Assessment iN Dry Eye (SANDE). The OSDI is a frequency-based questionnaire that contains 3 ocular symptom questions, 6 vision-related function questions, and 3 environmental trigger questions; SPEED and SANDE evaluated subjects on the frequency and severity of symptoms via 8 questions, and 2 visual analogue scales, respectively. This study adopted the Asia Dry Eye Society's criteria of an OSDI score $\geq 13$ and a strict tear break-up time (TBUT) threshold of $\leq 5$ seconds as being clinically significant. ${ }^{5}$ For all study patients, their basic demographic information such as age and gender, and diabetes-related clinical parameters such as serum glycated haemoglobin (HbA1c), duration of diabetes, BMI, fasting blood glucose, total cholesterol, low-density lipoprotein cholesterol (LDL-C), high-density lipoprotein 
cholesterol (HDL-C), triglyceride, creatinine, and estimated-glomerular filtration rate (eGFR) were obtained from questionnaires, and electronic patient records, respectively. $\mathrm{HbA1C}$ was measured every 3 to 6 months during follow-up, in whole blood using cation-exchange HPLC on Bio-Rad Variant (Bio-Rad Labs, CA, USA). The assay was certified by National Glycohemoglobin Standardization Program and standardized to Diabetes Control and Complications Trial reference assay.

\section{Statistical analysis}

All statistical analyses were performed using SPSS Statistics software (version 22.0). Descriptive statistics were used to summarise the demographic and clinical data. For a multivariable regression analysis with 12 predictors, given a desired probability level of 0.05 , a desired statistical power of 0.8 , and an anticipated effect size $\left(f^{2}\right)$ of 0.35 , the minimum required sample size was estimated to be 61 for this study. The KolmogorovSmirnov test was used to determine the normality of the sample data. Non-parametric analysis was used for data that were not normally distributed. Values were reported as means \pm standard deviation (SD) or percentages, as appropriate. The $95 \%$ confidence intervals for proportions were computed using the exact binomial calculation. Correlation analysis (Pearson or Spearman) was used to determine the relationship between right-side average NITBUT or OSDI and the different independent variables such as age, gender, HbA1c, duration of diabetes, BMI, fasting blood glucose, total cholesterol, LDL-C, HDL-C, triglyceride, creatinine, and eGFR. The effects of each independent variable were estimated with multiple stepwise linear regression. The level of significance was set at $a=0.05$. Linear trend analysis with one-way ANOVA was performed for any significant independent variable(s) and the outcome measure(s). To calculate the age-adjusted prevalence rate of DES, the age-specific (crude) prevalence rates for each age group in the study were multiplied by their respective weights 
(proportions of type II DM patients in the corresponding age groups in Hong Kong). The summation of these products yielded the age-adjusted prevalence rate. The sexspecific and age-adjusted prevalence rates were obtained in the same way after stratification of the sample data by sex. The odds ratios of DES for any significant independent variable(s) were obtained from binary logistic regression.

\section{RESULTS}

The clinical characteristics of the study population are summarised in Table 1. The mean age was $65 \pm 11$ years old, $58 \%$ were male and $48 \%$ were of working age. There were 80 subjects with T2DM, whose mean duration of diabetes (from diagnosis) was 17 \pm 10 years. Their mean $\mathrm{HbA1c}$ was $7.6 \pm 1.5 \%$. Among the various eye-specific parameters measured, the mean OSDI coefficient was $16 \pm 18$, while the mean NITBUT of the right eyes was $7.1 \pm 2.7$ seconds. Of the 80 participants, 16 were clinically significant (An OSDI score $\geq 13$ and a strict NITBUT threshold of $\leq 5$ seconds), giving rise to an estimated age-adjusted prevalence of clinically significant tear film instability in our study population of $20 \%$ (95\% CI: $12-30 \%$ ) (Table 2). Table 3 illustrates the proportions of patients with T2DM who are both symptomatic for dry eye and show NITBUT of $<=5,<=7.5$ or $<=10$ s.

Pearson's correlation analysis revealed that five of the independent variables had a significant correlation with NITBUT. In addition to HbA1c $(r=-0.34, P=0.004)$, age $(r=-$ $0.28, P=0.015)$, duration of diabetes $(r=-0.26, P=0.022), H D L-C(r=0.25, P=0.03)$, and eGFR ( $r=0.23, P=0.04)$ also showed modest but significant correlations with NITBUT (Table 4). Figure 2 shows the scatterplot illustrating the relationship between HbA1c and NITBUT. Similar analysis was done for OSDI coefficient, but only HbA1c 
$(r=0.26, P=0.026)$ showed significant correlation. Stepwise multiple linear regression analysis for NITBUT revealed that HbA1c was the only independent variable associated with NITBUT (Table 5), and HbA1c alone accounted for $11 \%$ of the variation in NITBUT $\left(R^{2}=0.11, P=0.008\right)$. To test for the linear relationship between HbA1c and NITBUT, one-way ANOVA was used which confirmed a significance difference $(p=0.002)$ between the means of NITBUT in HbA1c quartiles, and a significant weighted linear contrast $(p=0.002)$. A similar analysis done for OSDI also showed that HbA1c as the only significant factor, which alone explained $8.7 \%$ of the variation in OSDI score $\left(R^{2}=0.087, P=0.018\right)$. Table 6 shows the mean values and standard deviations of NITBUT and OSDI scores by HbA1c quartiles. The odds of DES for increasing quartiles of HbA1c was obtained from binary logistic regression, adjusted for age and sex, which revealed that for every increase in $\mathrm{HbA1c}$ quartile, there is an $86 \%$ increased risk of having DES (odds ratio $=1.86,95 \% \mathrm{CI}(1.06,3.25), \mathrm{p}=0.03)$. Alternatively, for every percentage increase in $\mathrm{HbA1C}$, there is a $49 \%$ increased risk of having $\mathrm{DES}$ (odds ratio $=1.49,95 \% \mathrm{CI}(1.03,2.17), \mathrm{p}=0.04)$.

With regard to the OSDI assessment, the most commonly reported ocular symptoms among the 16 subjects who satisfied the Asia dry eye society's criteria (an OSDI score $\geq 13$ and a strict TBUT threshold of $\leq 5$ seconds) for a diagnosis of DES were: blurred vision (75\%), exacerbation of symptoms in windy or dry environments (75\%), poor vision (69\%), and limitation in reading (69\%). The additional questionnaires used in this study, SPEED and SANDE, were significantly correlated with each other $(r=0.20, p$ $=0.09)$ However, spearman's correlation analysis for OSDI and SPEED $(r=0.20, p=$ $0.088)$ or SANDE $(r=0.035, p=0.77)$ did not reveal any significant trends.

\section{DISCUSSION}


Up to $20 \%$ of our patients with T2DM suffers from symptomatic tear film instability. This is in contrast to the general population with only $7 \%$ suffering from dry eye syndrome, as shown in a previous study in Chinese subjects using similar definitions. ${ }^{6}$ More importantly, analysis of the systemic risk factors revealed a significant inverse correlation between serum HbA1c level and non-invasive tear break-up time, indicating that poor glycaemic control is independently associated with an unstable tear film, after adjusting for possible confounders. A significant positive correlation was also found between $\mathrm{HbA1c}$ and OSDI score, illustrating that patients with high $\mathrm{HbA1c}$ level are more likely to experience dry eye symptoms. Collectively, our results suggest that poor glycaemic control is an important contributing factor to tear film instability - a finding that is in accordance with the current understanding of the pathophysiological impact of diabetes on the ocular surface. ${ }^{29}$ This highlighted the link between systemic risk factors, dry eye symptoms and ocular tear film metrics. Our findings on the associations of $\mathrm{HbA1C}$ with signs and symptoms of DES are in agreement with published studies in other populations. ${ }^{26,30,31}$ In an ophthalmic specialist outpatient clinic-based study on 40 patients with T2DM, Bal et al. observed a significant decrease in TBUT and Schirmer's test values with higher HbA1c levels on univariate analysis, suggesting that poor glycaemic control may result in tear film dysfunction. ${ }^{30}$ Moreover, Kaiserman et al. found that the frequency of artificial teardrop use in patients with T2DM increases proportionately in correlation to HbA1c level, which reflects the possible role of glycaemic control on disease severity. ${ }^{3}$ The association between HbA1c and DES carries important clinical implications. First, while topical lubricating eyedrops remain the cornerstone for dry eye treatment, optimization of glycaemic control should also be an important consideration for achieving symptom relief in patients with T2DM and dry eye. ${ }^{3,30}$ Furthermore, in addition to the current practice of addressing DES only when 
patients complain of dry eye symptoms, regular ocular assessments for dry eye should be implemented in the routine follow-up appointments in patients with poor glycaemic control.

Screening for symptomatic DES in the clinic can be done by widely-available questionnaires, for instance, the OSDI, which has been previously validated for DES diagnosis in an Asian population. ${ }^{32}$ In our study, we found that most of our patients with T2DM who had DES complained of symptoms of blurred vision, exacerbation of symptoms in windy or dry environments, poor vision, and limitation in reading. Nonophthalmic physicians should be educated on these common symptoms of DES, since early awareness of DES symptoms can lead to earlier detection and treatment. Thus far, although the relatively simpler formats of newer symptom questionnaires, such as SPEED and SANDE, may also serve as more time efficient methods for DES screening, only the OSDI symptom score has been validated for use in dry eye diagnosis in Asian populations. ${ }^{33,34}$ In our study, there was no significant correlation between OSDI score and both SPEED or SANDE. Furthermore, those frequent dry eye symptoms reported among our study patients were related to vision-related functions or environmental triggers, which are only considered by OSDI but not by SPEED or SANDE. Therefore, our study suggested that the OSDI might be the most optimal questionnaire for DES screening in Chinese patients with T2DM.

Our study have several limitations. First is the lack of control subjects without T2DM, which prevented us from making direct comparison on the prevalence of dry eye symptoms and tear film instability between the two groups. Second, our sample size of 80 is relatively small which might account for the wide $95 \%$ confidence intervals. Third, the cross-sectional design of our study might be limited to study the casual relationship 
among metabolic parameters, glycaemic control and DES. Furthermore, as patient recruitment was limited to the outpatient setting, the true prevalence of DES in T2DM may have been underestimated in our study. Moreover, the study focused on noninvasive tear film metrics, and as such Schirmer's test, corneal staining and tear osmolarity, which could provide further information regarding the severity and type of dry eye disease, were not included. Last but not least, there was no information regarding the living habits of the patients, such as the duration of usage of electronic devices, the use of lubricating eyedrops, the use of contact lenses, oral supplements or diabetic medications, outdoor exposure, dietary practice, sleep duration, as well as the educational level and occupation, which might all be potential confounders for the outcome, although lubricant eyedrop usage was particularly difficult to quantify due to major differences in lubricant type, constituents and drop volume between products and patients.

Our study, using questionnaires and non-invasive tear film metrics, had estimated the prevalence of probable DES amongst Chinese patients with T2DM to be high at $20 \%$, and revealed $\mathrm{HbA1C}$ as a significant predictor of tear film instability and dry eye symptoms. This highlights the importance of long-term blood glucose as a modifiable risk factor for symptomatic dry eye disease in T2DM. Hence, for physicians treating patients with T2DM, we recommend the implementation of early screening and management of dry eye, using the OSDI questionnaire, in routine follow-up appointments, especially for patients with poorly-controlled blood glucose. For ophthalmologists treating patients with diabetes-related dry eye, we recommend the monitoring and control of $\mathrm{HbA} 1 \mathrm{c}$ as an essential part of patient management.

\section{Acknowledgments}


The School of Public Health, Li Ka Shing Faculty of Medicine, University of Hong Kong, provided assistance in coordinating this project. The Oculus Keratograph 5M device, for dry eye measurements conducted in this project, was generously donated by the Shih Co Charitable Foundation.

\section{REFERENCES}

1. Quan J, Li TK, Pang H, et al. Diabetes incidence and prevalence in Hong Kong, China during 2006-2014. Diabet Med 2017; 34(7):902-908.

2. Bhavsar AS, Bhavsar SG, Jain SM. A review on recent advances in dry eye: Pathogenesis and management. Oman J Ophthalmo/2011;4(2):50-6.

3. Kaiserman I, Kaiserman N, Nakar S, Vinker S. Dry eye in diabetic patients. Am J Ophthalmol 2005; 139(3):498-503.

4. Tavakoli M, Kallinikos PA, Efron N, Boulton AJ, Malik RA. Corneal sensitivity is reduced and relates to the severity of neuropathy in patients with diabetes. Diabetes Care 2007;30(7):1895-7 .

5. Tsubota K, Yokoi N, Shimazaki J, et al. New Perspectives on Dry Eye Definition and Diagnosis: A Consensus Report by the Asia Dry Eye Society. Ocul Surf 2017; 15(1):65-76.

6. Lam DKT, Wong VWY, Chow VWS, Chi SCC. Epidemiology of dry eye syndrome in Hong Kong: a cross-sectional population-based study. Hong Kong $\mathrm{J}$ Ophthalamol 2011; 15:58-62.

7. Schaumberg DA, Gulati A, Mathers WD, et al. Development and validation of a short global dry eye symptom index. Ocul Surf 2007; 5(1):50-7. 
8. Yu J, Asche CV, Fairchild $\mathrm{CJ}$. The economic burden of dry eye disease in the United States: a decision tree analysis. Cornea 2011; 30(4):379-87.

9. Buchholz P, Steeds CS, Stern LS, et al. Utility assessment to measure the impact of dry eye disease. Ocul Surf 2006; 4:155-61.

10.Schiffman RM, Walt JG, Jacobsen G, Doyle JJ, Lebovics G, Sumner W. Utility assessment among patients with dry eye disease. Ophthalmology 2003; 110:1412-9.

11. Manaviat MR, Rashidi M, Afkhami-Ardekani M, Shoja MR. Prevalence of dry eye syndrome and diabetic retinopathy in type 2 diabetic patients. BMC Ophthalmol 2008; 8:10.

12. Burda N, Mema V, Mahmudi E, et al. Prevalence of dry eye syndrome at patients with diabetus melitus tip 2, one year retrospective study May 2011-June 2012. Journal of Acute Disease 2012;1(2):110-114

13. Wong KC, Wang Z. Prevalence of type 2 diabetes mellitus of Chinese populations in Mainland China, Hong Kong, and Taiwan. Diabetes Res Clin Pract 2006;73(2):126-34

14. Yoon $\mathrm{KH}$, Lee JH, Kim JW, et al. Epidemic obesity and type 2 diabetes in Asia. Lancet 2006;368(9548):1681-8.

15. Nyamdorj R, Pitkäniemi J, Tuomilehto J, et al. Ethnic comparison of the association of undiagnosed diabetes with obesity. Int J Obes (Lond) 2010;34(2):332-9.

16. Chan JC, Malik V, Jia W, et al. Diabetes in Asia: epidemiology, risk factors, and pathophysiology. JAMA 2009;301(20):2129-40.

17. Threatt J, Williamson JF, Huynh K, Davis RM. Ocular Disease, Knowledge, and Technology Applications in Patients with Diabetes. Am J Med Sci 2013;345(4):266-70. 
18. Goebbels M. Tear secretion and tear film function in insulin dependent diabetics. $\mathrm{Br} J$ Ophthalmo/2000; 84(1): 19-21.

19. Dogru M, Katakami C, Inoue M. Tear function and ocular surface changes in noninsulin-dependent diabetes mellitus. Ophthalmology 2001;108(3):586-92.

20. Figueroa-Ortiz LC, Jiménez Rodríguez E, García-Ben A, García-Campos J. Study of tear function and the conjunctival surface in diabetic patients. Arch Soc Esp Oftalmol 2011;86(4):107-12.

21. Cousen P, Cackett P, Bennett H, Swa K, Dhillon B. Tear production and corneal sensitivity in diabetes. J Diabetes Complications 2007;21(6):371-3.

22. Achtsidis V, Eleftheriadou I, Kozanidou E, et al. Dry eye syndrome in subjects with diabetes and association with neuropathy. Diabetes Care 2014;37(10):e2101.

23. Bettahi I, Sun H, Gao N, et al. Genome-wide transcriptional analysis of differentially expressed genes in diabetic, healing corneal epithelial cells: hyperglycemia-suppressed TGF $\beta 3$ expression contributes to the delay of epithelial wound healing in diabetic corneas. Diabetes 2014;63(2):715-27.

24. Wakuta M, Morishige N, Chikama T, Seki K, Nagano T, Nishida T. Delayed wound closure and phenotypic changes in corneal epithelium of the spontaneously diabetic Goto-Kakizaki rat. Invest Ophthalmol Vis Sci 2007;48(2):590-6.

25. Shaikh R. Prevalence of dry eye disease in type 2 diabetic patients and its correlation with the duration, glycemic control and retinopathy. A/ Almeen J Med Sci 2015; 8(3):225-229.

26. Seifart U, Strempel I. The dry eye syndrome and diabetes mellitus. Ophthalmologe 1994; 91(2):235-239. 
27. Xu KP1, Li Y, Ljubimov AV, Yu FS. High Glucose Suppresses Epidermal Growth Factor Receptor/Phosphatidylinositol 3-Kinase/Akt Signaling Pathway and Attenuates Corneal Epithelial Wound Healing. Diabetes 2009; 58(5):1077-85.

28. Uchino M, Yokoi N, Uchino Y, et al. Prevalence of dry eye disease and its risk factors in visual display terminal users: the Osaka study. Am J Ophthalmol 2013;156(4):759-66

29. Shih KC, Lam KS, Tong L. A systematic review on the impact of diabetes mellitus on the ocular surface. Nutr Diabetes 2017; 7(3):e251.

30. Bal C, Mukherjee S, Dan S. Effect of Long Term Glycemic Control on Precorneal Tear Film in Diabetic Patients. IOSR-JDMS 2014 Nov; 13(11):51-53.

31. Najafi $L$, Malek M, Valojerdi $A E$, et al. Dry eye and its correlation to diabetes microvascular complications in people with type 2 diabetes mellitus. $J$ Diabetes Complications $2013 ; 27(5): 459-62$

32. Li M, Gong L, Chapin WJ, Zhu M. Assessment of vision-related quality of life in dry eye patients. Invest Ophthalmol Vis Sci 2012; 53(9):5722-7.

33. Asiedu K, Kyei S, Mensah SN, Ocansey S, Abu LS, Kyere EA. Ocular Surface Disease Index (OSDI) Versus the Standard Patient Evaluation of Eye Dryness (SPEED): A Study of a Nonclinical Sample. Cornea 2016; 35(2):175-80.

34. Paulsen AJ, Cruickshanks KJ, Fischer ME, et al. Dry eye in the beaver dam offspring study: prevalence, risk factors, and health-related quality of life. $A m \mathrm{~J}$ Ophthalmol2014; 157(4):799-806. 


\section{TABLES}

Table 1: Baseline characteristics of the study population

\begin{tabular}{lc}
\hline Characteristics & $\begin{array}{c}(\boldsymbol{n}=\mathbf{8 0}) \\
(\text { mean, SD) }\end{array}$ \\
\hline $\begin{array}{l}\text { Demographics } \\
\text { Age (years) }\end{array}$ & $65 \pm 11$ \\
Male, no. (\%) & 58 \\
Clinical parameters & \\
DM duration (years) & $17 \pm 10$ \\
HbA1c (\%) & $7.6 \pm 1.5$ \\
Fasting blood glucose & \\
(mmol/L) & $8.0 \pm 2.7$ \\
BMI (kg/m ${ }^{2}$ ) & $26 \pm 4.3$ \\
Total Cholesterol & \\
(mmol/L) & $3.9 \pm 0.80$ \\
LDL-C (mmol/L) & $1.98 \pm 0.68$ \\
HDL-C (mmol/L) & $1.24 \pm 0.36$ \\
TG (mmol/L) & $1.57 \pm 0.95$ \\
Creatinine $(\mu \mathrm{mol} / \mathrm{L})$ & $104 \pm 102$ \\
eGFR (mL/min/1.73 $\mathrm{m}^{2}$ ) & $72 \pm 25$ \\
Eye-specific & \\
parameters & \\
OSDI score & \\
SANDE score & $16 \pm 18$ \\
SPEED score & $23 \pm 26$ \\
NITBUT (Right) $(\mathrm{s})$ & $5.7 \pm 5.2$ \\
NITBUT (Left) $(\mathrm{s})$ & $7.5 \pm 2.7$ \\
\hline
\end{tabular}

Abbreviations: Diabetes mellitus (DM); Body mass index (BMI); Low-density lipoprotein cholesterol (LDL-C); High-density lipoprotein cholesterol (HDL-C); Triglyceride (TG); Estimated-glomerular filtration rate (eGFR); Non-invasive tear break-up time (NITBUT). 
Table 2: Crude and age-adjusted prevalence rates of probable DES among Chinese type II diabetic subjects by sex and age groups.

\begin{tabular}{|c|c|c|c|c|c|c|}
\hline $\begin{array}{l}\text { Age } \\
\text { Group }\end{array}$ & $\begin{array}{l}\text { n (Age } \\
\text { group) }\end{array}$ & $\begin{array}{c}\text { n (Probable } \\
\text { DES) }\end{array}$ & $\begin{array}{c}\text { Crude } \\
\text { Prevalence } \\
\text { rate }\end{array}$ & $95 \% \mathrm{CI}$ & $\begin{array}{c}\text { Weig } \\
\text { ht }\end{array}$ & $\begin{array}{c}\text { Weighted } \\
\text { Prevalence } \\
\text { rate }\end{array}$ \\
\hline $\begin{array}{l}\text { Both } \\
\text { sexes }\end{array}$ & & & & & & \\
\hline$<50$ & 3 & 0 & 0.00 & $\begin{array}{l}0.00 \\
0.71 \\
0.05\end{array}$ & 0.17 & 0.00 \\
\hline 50-59 & 22 & 5 & 0.23 & $\begin{array}{l}0.41 \\
0.12\end{array}$ & 0.19 & 0.04 \\
\hline $60-69$ & 26 & 7 & 0.27 & $\begin{array}{l}0.48 \\
0.06,\end{array}$ & 0.42 & 0.11 \\
\hline 70-79 & 19 & 4 & 0.21 & $\begin{array}{l}0.46 \\
0.12\end{array}$ & 0.22 & 0.05 \\
\hline $\begin{array}{l}\text { Total } \\
\text { Male }\end{array}$ & 80 & 16 & 0.20 & 0.30 & 1.00 & 0.20 \\
\hline$<50$ & 2 & 0 & 0.00 & $\begin{array}{l}0.00 \\
0.84 \\
0.00,\end{array}$ & & 0.00 \\
\hline $50-59$ & 13 & 1 & 0.08 & $\begin{array}{l}0.36 \\
0.00,\end{array}$ & 0.19 & 0.01 \\
\hline $60-69$ & 11 & 1 & 0.09 & $\begin{array}{l}0.41 \\
0.09,\end{array}$ & 0.43 & 0.04 \\
\hline 70-79 & 13 & 4 & 0.31 & $\begin{array}{l}0.61 \\
0.05,\end{array}$ & 0.23 & 0.07 \\
\hline $\begin{array}{l}\text { Total } \\
\text { Femal } \\
\text { e }\end{array}$ & 46 & 6 & 0.13 & 0.26 & 1.00 & 0.12 \\
\hline$<50$ & 1 & 0 & 0.00 & 0.00 & & \\
\hline
\end{tabular}

This article is protected by copyright. All rights reserved. 


\begin{tabular}{|c|c|c|c|c|c|c|}
\hline & & & & 0.98 & & \\
\hline 50-59 & 9 & 4 & 0.44 & $\begin{array}{c}0.14 \\
0.79 \\
0.16\end{array}$ & 0.23 & 0.10 \\
\hline $60-69$ & 15 & 6 & 0.40 & $\begin{array}{l}0.67 \\
0.00\end{array}$ & 0.50 & 0.20 \\
\hline 70-79 & 6 & 0 & 0.00 & $\begin{array}{l}0.46 \\
0.15\end{array}$ & 0.03 & 0.00 \\
\hline Total & 34 & 10 & 0.29 & 0.47 & 1.00 & 0.30 \\
\hline
\end{tabular}

Abbreviation: Dry eye syndrome (DES); Confidence interval (CI).

Table 3: Proportions of diabetic subjects who are both symptomatic for dry eye and show NITBUT of $<5,<7.5$, or $<10$ s.

\begin{tabular}{lc}
\hline & Symptomatic for dry eye (OSDI $\geq 13)$ \\
\cline { 2 - 2 } NITBUT (s) & Proportions of diabetics $(95 \% \mathrm{CI})$ \\
\hline$<5$ & $0.20(0.12,0.30)$ \\
$<7.5$ & $0.29(0.19,0.39)$ \\
$<10$ & $0.40(0.30,0.51)$ \\
\hline
\end{tabular}

Abbreviations: Non-invasive tear break-up time (NITBUT); Ocular Surface Disease Index (OSDI); Confidence interval (CI).

Table 4: Pearson and Spearman correlation of independent variables with NITBUT and OSDI, respectively, among Chinese type II diabetic subjects.

\begin{tabular}{ccccccc}
\hline & \multicolumn{2}{c}{ NITBUT } & & \multicolumn{2}{c}{ OSDI } \\
\cline { 2 - 3 } \cline { 5 - 7 } & & value & & & & P-value \\
\hline
\end{tabular}

This article is protected by copyright. All rights reserved. 


\begin{tabular}{|c|c|c|c|c|}
\hline & & 0.01 & & \\
\hline Age & -0.28 & 5 & 0.10 & 0.40 \\
\hline Gender & -0.03 & $\begin{array}{l}0.40 \\
\mathbf{0 . 0 2}\end{array}$ & 0.11 & 0.37 \\
\hline Duration of DM & -0.26 & 2 & $\begin{array}{c}0.09 \\
-\end{array}$ & 0.45 \\
\hline BMI & -0.01 & $\begin{array}{l}0.46 \\
0.00\end{array}$ & 0.07 & 0.57 \\
\hline $\begin{array}{l}\text { HbA1c } \\
\text { Fasting blood }\end{array}$ & -0.34 & 4 & $\begin{array}{l}0.26 \\
0.14\end{array}$ & 0.026 \\
\hline glucose & -0.19 & 0.07 & $\begin{array}{l}6 \\
-\end{array}$ & 0.22 \\
\hline Total Cholesterol & 0.03 & 0.42 & 0.04 & 0.76 \\
\hline LDL-C & -0.14 & 0.13 & 0.03 & 0.82 \\
\hline HDL-C & 0.25 & 0.03 & $\begin{array}{c}0.18 \\
-\end{array}$ & 0.13 \\
\hline TG & 0.06 & 0.33 & $\begin{array}{c}0.01 \\
-\end{array}$ & 0.91 \\
\hline Creatinine & -0.04 & 0.37 & 0.19 & 0.10 \\
\hline eGFR & 0.23 & 0.04 & 0.01 & 0.92 \\
\hline
\end{tabular}

Abbreviations: Non-invasive tear break-up time (NITBUT); Ocular Surface Disease Index (OSDI); Body mass index (BMI); Low-density lipoprotein cholesterol (LDL-C); High- density lipoprotein cholesterol (HDL-C); Triglyceride (TG); Estimated-glomerular filtration rate (eGFR).

Table 5: Results of stepwise multiple linear regression analysis of NITBUT and OSDI coefficient among Chinese type II diabetic subjects.

\begin{tabular}{ccccccc}
\hline & \multicolumn{3}{c}{ NITBUT } & & \multicolumn{3}{c}{ OSDI } \\
\cline { 2 - 6 } & $t$ & P-value & & $t$ & $\mathrm{P}-$ \\
\hline
\end{tabular}




\begin{tabular}{|c|c|c|c|c|c|c|}
\hline & & & & & & value \\
\hline Age & -.019 & 1.46 & 0.15 & 0.03 & 0.23 & 0.82 \\
\hline Gender & -0.06 & 0.49 & 0.63 & 0.03 & 0.23 & 0.82 \\
\hline Duration of DM & -0.16 & 1.24 & 0.22 & 0.04 & $\begin{array}{c}0.29 \\
-\end{array}$ & 0.78 \\
\hline BMI & 0.01 & $\begin{array}{c}0.07 \\
-\end{array}$ & 0.94 & -0.04 & 0.32 & 0.75 \\
\hline $\begin{array}{l}\text { HbA1c } \\
\text { Fasting blood }\end{array}$ & -0.34 & 2.73 & 0.008 & 0.30 & $\begin{array}{c}2.43 \\
-\end{array}$ & 0.018 \\
\hline glucose & 0.03 & 0.16 & 0.87 & -0.04 & 0.28 & 0.78 \\
\hline Total Cholesterol & -0.04 & $\begin{array}{c}0.33 \\
-\end{array}$ & 0.74 & 0.17 & 1.38 & 0.17 \\
\hline LDL-C & -0.17 & 1.40 & 0.17 & 0.22 & 1.87 & 0.07 \\
\hline HDL-C & 0.20 & 1.67 & 0.10 & -0.12 & 1.00 & 0.32 \\
\hline TG & 0.02 & 0.17 & 0.97 & -0.05 & $\begin{array}{c}0.37 \\
-\end{array}$ & 0.72 \\
\hline Creatinine & 0.04 & 0.35 & 0.73 & -0.03 & 0.25 & 0.81 \\
\hline eGFR & 0.14 & 1.11 & 0.27 & 0.08 & 0.63 & 0.53 \\
\hline
\end{tabular}

Abbreviations: Non-invasive tear break-up time (NITBUT); Ocular Surface Disease Index (OSDI); Body mass index (BMI); Low-density lipoprotein cholesterol (LDL-C); High- density lipoprotein cholesterol (HDL-C); Triglyceride (TG); Estimatedglomerular filtration rate (eGFR).

Table 6: The mean values and standard deviations of NITBUT and OSDI by HbA1c quartiles. 


\begin{tabular}{cccccccccc}
\hline \multicolumn{3}{c}{ HbA1C } & & & \multicolumn{2}{c}{ NITBUT } & & \multicolumn{2}{c}{ OSDI } \\
\cline { 1 - 2 } \cline { 8 - 9 } Quartiles & Minimum & Maximum & & mean & $(\mathrm{s})$ & SD & & mean & SD \\
\hline $1^{\text {st }}$ & 5.00 & 6.73 & & 9.22 & 2.56 & & 15 & 14 \\
$2^{\text {nd }}$ & 6.74 & 7.35 & & 6.44 & 2.88 & & 12 & 12 \\
$3^{\text {rd }}$ & 7.36 & 8.38 & & 6.74 & 2.04 & & 20 & 19 \\
$4^{\text {th }}$ & 8.39 & 13 & & 6.38 & 2.09 & & 17 & 17 \\
\hline
\end{tabular}

Abbreviations: Non-invasive tear break-up time (NITBUT); Ocular Surface Disease Index (OSDI); Standard deviation (SD). 


\section{FIGURES}

Figure 1: Flowchart illustration of the study procedure.

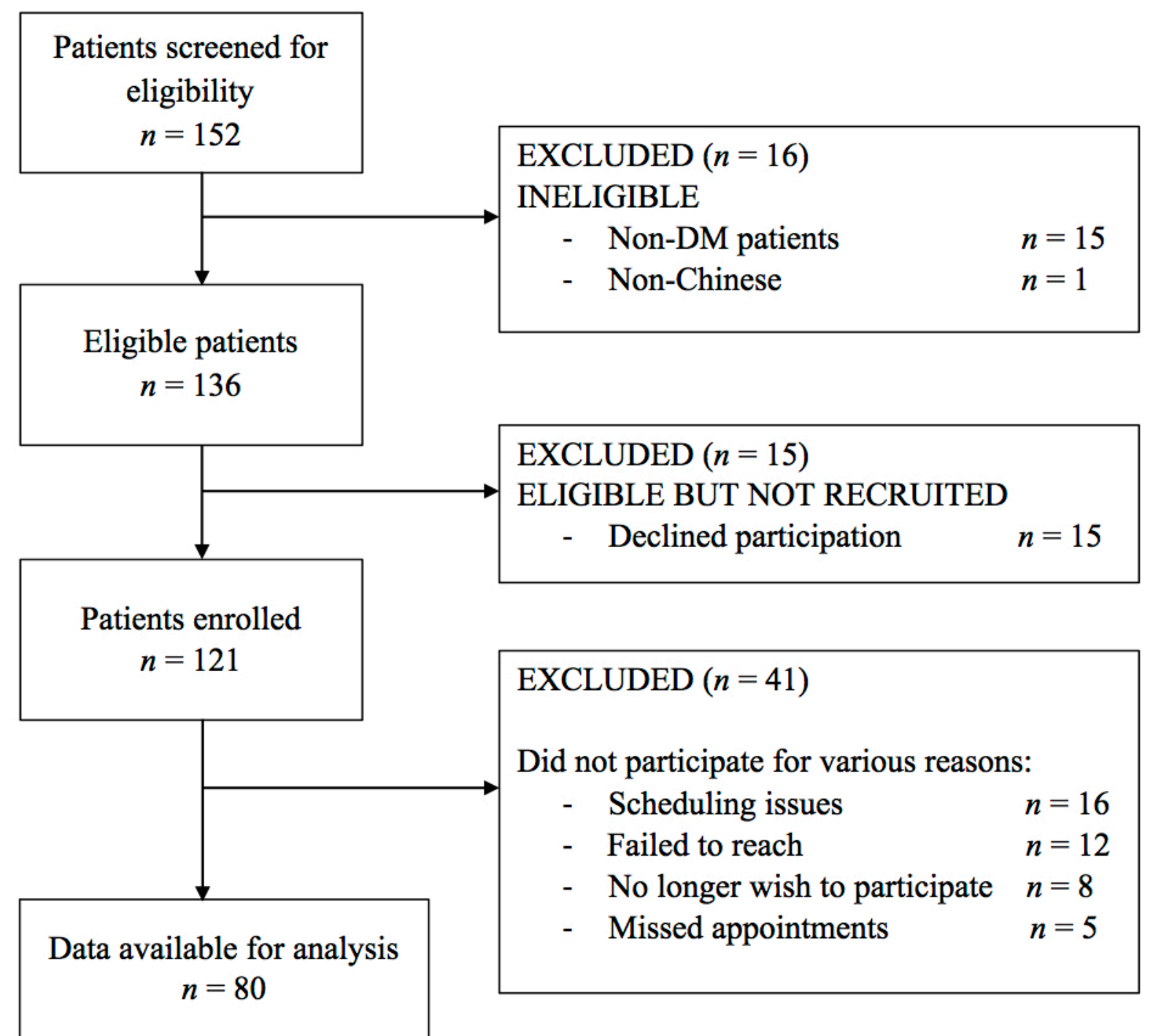

This article is protected by copyright. All rights reserved. 
Figure 2: Scatterplot of HbA1c and NITBUT

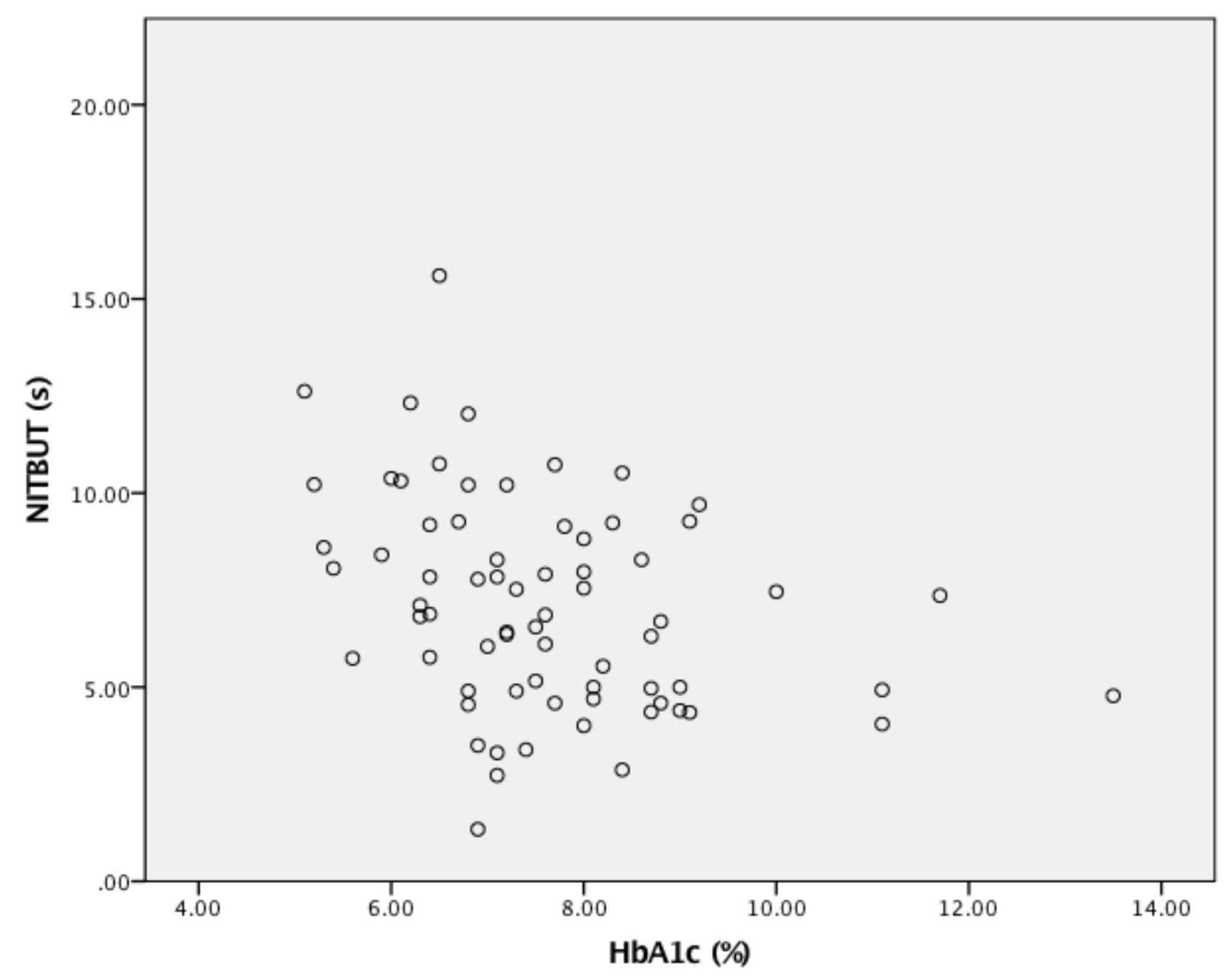

This article is protected by copyright. All rights reserved. 


\section{University Library}

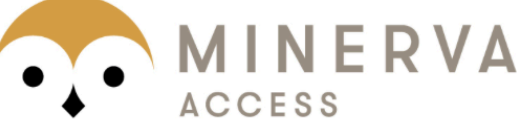

A gateway to Melbourne's research publications

Minerva Access is the Institutional Repository of The University of Melbourne

Author/s:

Ma, A;Mak, MSY;Shih, KC;Tsui, CKY;Cheung, RKY;Lee, SH;Leung, H;Leung, JNS;Leung,

JTH;Van-Boswell, MZ;Wong, MTL;Ng, AL-K;Lee, C-H;Jhanji, V;Tong, L

Title:

Association of long-term glycaemic control on tear break-up times and dry eye symptoms in Chinese patients with type 2 diabetes

Date:

2018-08-01

Citation:

Ma, A., Mak, M. S. Y., Shih, K. C., Tsui, C. K. Y., Cheung, R. K. Y., Lee, S. H., Leung, H., Leung, J. N. S., Leung, J. T. H., Van-Boswell, M. Z., Wong, M. T. L., Ng, A. L. -K., Lee, C. H., Jhanji, V. \& Tong, L. (2018). Association of long-term glycaemic control on tear breakup times and dry eye symptoms in Chinese patients with type 2 diabetes. CLINICAL AND EXPERIMENTAL OPHTHALMOLOGY, 46 (6), pp.608-615. https://doi.org/10.1111/ceo.13146.

Persistent Link:

http://hdl.handle.net/11343/283541 